\title{
The Children's Delphi: considerations for developing a programme for promoting children's self-concept and well- being
}

\author{
Elizabeth Benninger and Shazly Savahl
}

\begin{abstract}
This study is premised on the notion that intervention programmes aimed at improving children's well-being should be inclusive of activities which promote children's self-concept. Using a child participation framework, this study aimed to explore children's perceptions of the nature and content of intervention programmes aimed at improving children's self-concept within two impoverished communities of the Western Cape, South Africa. The Delphi technique was followed with a group of 10 children between the ages of 10 and 12 years who were considered to be knowledgeable experts and authorities on matters affecting their lives and well-being of children. They suggested that intervention programmes include a focus on safety, the provision of social support, the creation of opportunities for learning and for play and the provision of basic material needs.
\end{abstract}

\section{Introduction}

The 'self' in social psychology is seen as a multidimensional, dynamic and complex construct, largely created as a reflection of children's interaction and engagement with their social environment (Kenny \& McEachern, 2009). It is, therefore, essentially shaped by social institutions, culture, history and the social context in which they live (Staub, 2003). Recent research (see Benninger \& Savahl, 2016a; Fattore, Mason \& Watson, 2007; Savahl et al., 2015; September \& Savahl, 2009) has shown that the ways in which children experience and make sense of the 'self' plays a central role in their psychosocial functioning and overall well-being. Subsequently, the importance of the self-concept has been foregrounded in intervention programmes aimed at improving children's well-being (Swann, Chang-Schneider, \&McClarty, 2007).

Prior studies have recommended that the self-concept be supported through intervention programmes which are inclusive of meaningful social relationships, feelings of inclusion, safety, coping skills, structured and unstructured activities, opportunities for reflection and through addressing the larger structural issues which may influence the resources available to nurture a healthy self-concept (see Demaray et al., 2009; McMurray et al., 2011; Noble-Carr et al., 2013; Rogers et al., 2012). An example is seen in a study conducted by McMurray (2010) with 13 British adolescents from a low socioeconomic background. The study found that the self-identity 
of the participants was shaped by their social relationships; they positioned the self in relation to their friends, siblings and extended family, and reported a need to build an emotional attachment with an adult figure. The study supported the need for intervention programmes to include activities which focus on the development of trusting relationships with peers and supportive adults and which link youth to services that assist them in the reconstruction of healthier self-identities.

Further evidence is presented by Demaray et al. (2009) who investigated the role that social support played on children's self-concept, specifically focused on the relationship between the perceived frequency and importance of social support. Participants with high close friend support had higher levels of self-concept than those with average or low levels. The results suggest that support from significant adults is a critical factor in the development of a positive self-concept. The authors considered implications for intervention programmes aimed at the promotion of a healthy self-concept to include assisting children to develop positive networks of social support, including support from peers, family, and other caring adults.

Research conducted by Rogers et al. (2012) described the role that social identity played in the development of children's self-concept in a culturally diverse sample of children from the United States. The meaning participants ascribed to their social identity connected to their ethnic group encompassed ideas such as language, physical appearance, pride, relative social position and culture. The findings of the study support the need for further interventions that are aimed at promoting a healthy self-concept to be inclusive of activities which nurture self-reflection and meaning-making processes.

Noble-Carr et al. (2013) explored the role of social identity and meaning-making in the lives of vulnerable adolescents in Australia who were enrolled in youth support services and the implications for programmes which support their needs. The study recommended the need for support services to emphasise and understand issues around young peoples' personal identity and to assist young people to understand the importance of making positive social connections. For example, services should focus their efforts on facilitating, building and promoting opportunities for young people to develop personal caring connections, meaningful connections to the community, a sense of belonging, mastery and hope.

Research conducted by Kenny \& McEachern (2009) amongst a racially diverse sample of children from the United States explored how a sense of social isolation may negatively contribute to the self-concept, specifically as it relates to feelings of self-worth. The study found the self-concept to be closely linked to the internalisation of prejudice and devaluation of one's racial minority group. The authors recommended that intervention programmes include the promotion of social inclusion and cultural competency.

The research by Swann et al. (2007) discussed the subjective perceptions of children to be an important aspect of their quality of life and a worthwhile focus for the development and implementation of theoretically informed programmes. The findings supported optimal interventions to focus directly on enhancing the self-concept in specific areas relevant to the goals of theintervention and to include opportunities for skills development. 
Although the existing studies provide valuable information for informing self-concept related intervention programmes for children from diverse social contexts, socio-economic circumstances and cultures, these implications are drawn upon by the researchers, who are viewed to be the 'experts' in their field of study. However, there remains a gap in the literature with regard to children's own perceptions and understandings of the nature and content of intervention programmes aimed to improve children's self-concept and well-being. The inclusion of children's perspectives on issues related to their well-being is increasingly supported by current child research, as stated by Casas (2016):

Children are slowly being recognized as key informants and competent informers on their own lives. Consequently, their voices, their evaluations and their points of view are increasingly more accepted as key sources of information in scientific research (p. 9).

Subsequently, this study is premised on the notion that intervention programmes aimed at improving children's well-being should take special consideration of activities which promote children's self-concept, and should be inclusive of the perspectives and recommendations of the children themselves. This study utilises the Delphi method with children, hereafter referred to as the Children's Delphi, to achieve this. The Children's Delphi is based on the epistemological position that children are knowledgeable experts and authorities on matters pertaining to children's lives and well-being. In the current study the Children's Delphi is conceptualised as panel of children, who through a prolonged engagement with the research process, provide in-depth knowledge and insight into the development of programmes for the promotion of a health selfconcept.

\section{Study aim}

Using the Children's Delphi method within a child participation framework, the study aimed to explore children's perspectives on developing intervention programmes for the promotion of a healthy self-concept within two urban impoverished communities of the Western Cape in South Africa.

\section{Child participation}

Child participation research places the child at the centre of the research process in order to gain a detailed understanding of their subjective experiences and meaning-making processes while taking into consideration the social, cultural and historical context (Fattore et al, 2007). Prior child participation research has contributed towards valuable knowledge around issues related to children's attitudes and experiences, such as issues around the environment, labour, schooling, play, gender, sexuality and spirituality (Mason \& Watson, 2014). Mason \& Watson (2014) point to child participation as a critical framework for the inclusion of children in research, which has formerly been defined by privileged and powerful adults. They further state that 'children typically have been at the bottom of the hierarchy of formal knowledge production, with their knowledge excluded or marginalized because they are outside the dominant knowledge production forums, including academic institutions' (p. 2757). Child participation research recognises children as actors and knowers who are knowledgeable about their lives (Fattore et al., 
2007). This epistemological position advanced by the theoretical and methodological assertions of the 'new sociology of childhood' has its genesis in international legislation on children's rights, mostly notably the United Nations Convention on the Rights of the Child (UNCRC). Article 12 of the UNCRC states that children have the right to freely express their opinion, to be heard and for their opinions to be taken seriously on all matters which affect them (United Nations General Assembly, 1989). Article 13 supports child participation in the form of freedom of expression. This includes the right to seek, receive or impart information and ideas through the child's preferred form of communication.

While children have the right to voice their needs, they also may need the support of adults to take them seriously and to assist them with access to community, organisational and structural resources. Often in the research process, the researcher's view of the child is based on societal beliefs, rather than the child's beliefs, with children rarely consulted to define their own problems and solutions (Langhout \& Thomas, 2010). It is argued that this approach to research disempowers children as adult 'experts' make decisions on their behalf without consulting them or including them in the decision-making process (Crivello et al., 2009, Prilleltensky, 2010). Through child participation research, collaborative methodologies are valued to ensure that children are involved in defining problems and solutions (Langhout \& Thomas, 2010). The researcher's role in child participation shifts from the adult-centric perspective, working instead as a collaborator in ways which enhance the power children have over their own lives (Langhout\& Thomas, 2010).

While using various research methods with children, it is important to note that a method is only participatory depending on the way in which it is used (Crivello et al., 2009). Children are often left out of participation processes in research or may participate to various degrees (Crivello et al., 2009). Moore et al. (2016) stress the importance of providing children with more power and choice in the research process and in demonstrating, through words and actions, the value of their expertise and viewpoints. For research to be fully participatory, the participants should be engaged in every stage of the research process. Children should take on the role of social actors, collaborators and co-researchers, and the adult researchers should collaborate in ways which enhance the power children have over their own lives (Langhout\& Thomas, 2010).

Child participation research has the potential for a range of benefits for the individual participants, their communities and the promotion of child well-being. Helping children to articulate their opinions on their environment is crucial for psychosocial problem prevention and the promotion of mental health (De Winter, 1999). Furthermore, allowing children to have a meaningful voice and active role in managing their environment is a resource for healthy development (De Winter et al., 1999). The subjective knowledge identified through child participation can be utilised to gain a deeper understanding of access barriers and to collaborate with children and community members in creating solutions which are culturally and socially relevant for addressing child mental health (Shattell et al., 2008). Through participation, children's selfefficacy can be strengthened along with an increase in self-confidence. This is achieved asthe resources ofchildrenare developed, while incorporating skill building, social support and identity development (Langhout \& Thomas, 2010). 


\section{Methods}

\section{Research context}

The research was conducted within two urban communities located in the Cape Flats region of Cape Town, South Africa. The communities were formed as a result of Apartheid's system of institutionalised racism (1948-1994), which segregated the South African society on the premise of 'white' superiority and privilege (Lockhat \& Van Neikerk, 2000). During this period, South African residents were divided into four distinct racial classifications and forced to live in racially designated areas, with 'Black' and 'Coloured' residents relocated to poorly resourced areas referred to as townships. The townships of Lavender Hill, a historically 'Coloured' community, and Khayelitsha, a historically 'Black' community, were selected in order to capture the diversity of the childhood experience within the Cape Flats. The children in these communities remain impacted by the aftermath of Apartheid, where conditions of poverty and violence continue to pose a threat to their daily health and wellbeing.

\section{Design}

The overall design of the study was participatory with various methods used to ground the research process in the values and principles of child participation. A critical component of the design was the development of a child reference group which consisted of 10 child participants, selected from the participating communities, who served as co-researchers and consultants for a larger research project which aimed to explore how children construct and assign meaning to the self within their communities (see Benninger, 2016). The engagement of children in reference groups is based on the belief that children are competent social actors and experts with valuable insights which may be different than adults (Moore et al, 2016). While research using participatory methodologies with children has grown in recent years, Moore et al. (2016) make the essential point that 'there has been little discussion within the research field as to how children and young people may meaningfully and actively participate in co-reflective activities such as reference groups (p. 2).' In this study, we propose the use of the Delphi technique as a means for meaningfully engaging the child reference group as the Delphi participants premised on the notion that they are the experts of their lives and experiences.

The Delphi is a technique which captures the collective opinions of experts who have a consultative role on a particular topic (De Villiers, De Villiers, \& Kent, 2005) and takes the form of an exploratory exercise conducted with the expert panel related to the phenomenon under exploration (Kennedy, 2004). The technique includes a series of forums or consultations which take place until a consensus is reached about a particular topic or problem. Open-ended questions are used for eliciting opinions related to the topic, with additional questions formed based on the feedback from the previous rounds (De Villiers et al., 2002). The traditional Delphi is characterised by the five features of anonymity, iteration, controlled feedback, statistical group response and stability in responses (Hanafin \& Brooks, 2005).

Epistemologically, the Delphi technique has its roots in both positivist and interpretivist epistemologies (Hanafin \& Brooks, 2005). Those working from a positivist stand-point argue that the Delphi technique is grounded in the ontological position of a single reality, while those from an 
interpretivists/social constructionist position argue for multiple realities. The key argument of the interpretivist/social constructionist is that the group consensus is achieved through an intersubjective iterative process. In the current study the latter epistemological position is adopted and works from the assumption that children, as knowledgeable experts, are creating consensus through a continuous process of interaction and feedback.

\section{Children's Delphi process}

The data collection proceeded through the following steps associated with the Delphi. The first step included the selection of the appropriate participants for the Delphi. According to Hasson, Keeney, \& McKenna (2000), the participants should be selected purposively to form a panel of informed experts who have knowledge of the subject or problem under investigation. While the Delphi panel traditionally consists of adult experts who are perceived to be the experts in their professional field related to the Delphi aim, current research supports the importance of children as key informants and competent informers around issues related to their lives (Casas, 2016).

In this study, the experts were chosen in accordance with a child participation framework, where children were viewed to be the most suited for the Delphi panel. Firstly, they were viewed to be the experts of their own lives, with valuable knowledge and a deep insight, understanding and experiences of being a young person within their communities. Secondly, they participated in all stages of the larger child participation research project, which provided them with substantial indepth knowledge with regard to the subjective experiences of other children within their respective communities.

The Delphi participants were selected from the two participating communities of Khayelitsha (three boys, two girls) and Lavender Hill (three girls, two boys) between the ages of 10 and 12 years. They were also the members of the child reference group of the larger research project which aimed to explore children's constructions of the self within their respective communities (see Benninger, 2016). Purposive sampling was used to select the participants from two community-based organisations where the researchers were involved, based on the criteria of being the appropriate age, being available for the weekly research sessions, and expressing a willingness to participate. The primary researcher spent one year volunteering with the participating organisations prior to the commencement of the research process in order to build rapport amongst the study participants and had the opportunity to speak directly with the children about the details of the research project. Children who expressed interest in the project were requested to register as volunteers with the organisations. The child reference group functioned as co-researchers and community consultants throughout the entire research process, including the focus group discussions (Benninger \& Savahl, 2016b), Photovoice and community mapping activities (Benninger \& Savahl, 2016b), and the Delphi which formed a part of a larger PhD study of the primary author.The current paper reports on thefinal Delphistage of the project.

The next step stipulated by Hasson et al. (2000) is to identify the resources and skills of the participants and the appropriate means of communication to use throughout the process. Given that the participants were children residing within communities with an under-resourced education system, the literacy of the participants varied; it was therefore decided to provide the 
participants with various means of communicating their ideas through the use of writing, drawing and verbal response.

Three sessions were held with each Delphi group. While the content of the sessions followed a structured format, an informal atmosphere of engagement was encouraged with food and refreshments provided during each session. The first session began with a series of open-ended questions related to the themes which emerged throughout the larger research study. The discussions specifically explored how the perspectives of children in their communities could contribute towards the design of interventions aimed at promoting a healthy self-concept. The following questions were included: How do children think and feel about themselves in your community? What makes them think or feel that way? What barriers did our research discover which prevented children from viewing themselves in a positive way? What is needed for children to develop a healthy self-concept in your community? What should a programme aimed at improving child self-concept look like? In addition to verbal responses, discussion feedback was also provided through the use of drawings.

After the completion of the first session, the next included a review of the items discussed in the prior session and provided the opportunity for amendments and changes. The researcher drew three separate sections on a large whiteboard labelled Photovoice and community maps, focus group discussions and intervention programme. All of the themes identified by the participants in the prior session were listed in the applicable category on the white board. The participants then had the opportunity to add in items by verbally explaining or writing their responses in the appropriate category. This process continued until consensus was reached amongst all of the participants.

The third session of the Delphi included the data analysis. Once consensus was reached by all of the participants, the data from the Delphi was compiled into a report using content analysis and distributed to the participants during the third session for their review. The report included the thematic categories and themes as identified by the participants in the previous round. Once the participants approved the report, a final draft was written and distributed to each member for final approval.

\section{Procedures and ethics}

The research was approved by the Senate Research Ethics Committee at the university where the researchers are based. Two local NGOs assisted with the process of participant recruitment. An initial session was held with the selected participants, introducing the aims of the study, expectations of participation and included a discussion of the key ethics principles of informed consent, voluntary participation, confidentiality, the freedom to withdraw without consequence and the academic use of the data. The participants and their guardians were provided with an information sheet which included the details of the study as well as consent forms for both the guardian and the participant. The Delphi sessions were conducted by the primary researcher at a local community centre after school hours. Permission was sought from the participants to audio record the sessions. Lastly, involving the participants in the post data analysis phase of a research project was an important component of the methodological and ethical considerations and ensured 
that the data was accurately presented (Moore et al., 2016). This was achieved within the third session of the Delphi process where the final report was presented to the participants, who approved the report after a series of discussions. Furthermore, the participants assisted with presentations which were conducted at various forums with community stakeholders.

\section{Findings}

The participants divided their responses into two thematic categories: (i) factors which influence children's self-concept; (ii) suggestions for intervention programmes. Within the first thematic category, the themes of feelings, children's reality and relationships emerged. Within the second thematic category, the four themes included; safety, social support, opportunities for learning and for fun, and the provision of basic needs.

\section{Factors which influence a child's self-concept}

The participants first identified feelings to play a role in the way in which children constructed and assigned meaning to the 'self.' The participants described the children in their communities to have a variety of feelings and emotions, which were dependent on their regular experiences within their environment. For example, many of the children often felt afraid as a result of the constant threat from the local gangsters. This was at times reflected in narratives wherein they expressed a view that they were not worthy of being kept safe. These feelings of lack of selfworth were also described to occur when the adults in the community did not take the time to listen to the things which were important to children. They believed that children felt 'bad about themselves' because they felt undervalued by adults.

Children's reality for young people who resided in their communities was also identified to play a role in the local children's self-constructions. This reality was described to be in conflict with their expectations of an ideal childhood reality, where children were able to experience safety, nurturance and an ability to play outside. The contrasting reality of the participants was depicted to be one of violence, either as a result of the gangsters and crime outside of the home, or through their personal experiences of abuse in the home. This led to a sense of self constructed around a fear for their safety. Children in their community did not feel safe walking to school, playing in the parks or even at times in their own homes. They explained that children would feel better about themselves if adults would treat them with patience and kindness, rather than passing disparaging comments and profanities towards children.

The participants also described the children's reality to be related to an unclean physical environment which made children feel bad about themselves. A lack of resources because of the conditions of poverty was additionally tied into the children's reality, where they felt that their basic needs often went unmet.

Relationships with family, friends and 'other people' were identified as factors that could influence the self-concept of the children in their communities. For the participants who had supportive relationships in the home, family played an important role in making the children feel valued and safe, despite the social context of violence and poverty. One participant explained 'children feel happy and good about themselves when they have the things they need and 
when their parents buy them nice things. When parents swear and hit their children, it makes the children feel bad about themselves' (Female, age 11). For the participants who felt they had an abusive home environment, there seemed to be a natural shift towards other sources of support outside of the home which helped them to maintain the integrity of the 'self'. For example, several participants mentioned feeling supported by a teacher at school or childcare worker at an after-school programme, while experiencing abuse and neglect in the home: 'The coaches are not rude to children. When children are rude they actually are going to help you and they make kids say sorry' (Female, age 10). Furthermore, relationships with friends were also perceived to make children feel good about themselves, especially if they treated others well, shared nice things and played fun games. However, it was pointed out that relationships with friends also had the potential to make children feel bad about themselves when they gossiped, fought and used foul language.

\section{Implications for intervention programmes}

Safety was identified to be an imperative for any children's programme or intervention aimed at promoting a healthy self-concept. The participants explained that safety was crucial for children in their community and that 'good programmes will make sure children are safe' (Female, age 10). Social support was the second theme identified as crucial for an intervention programme. This support should be in the form of relationships with elders and peers. The participants described that children need adults for supervision, to play with, to talk to about their problems, to teach them to respect each other and to give advice: 'Children need adults who you can talk your problems to, and if someone did something to you at school, you can tell an adult, and if something happened to you, you can run to an adult and tell what happened to you (Female, age 11).' Relationships with other peers were identified as important, especially if these were formed around positive activities and behaviours. Having friends to participate in positive activities with was also identified as important for the development of a self-concept constructed around prosocial behaviours, rather than one around crime and other unhealthy behaviours such as drugs and alcohol use.

The next essential component identified by the participants as essential for intervention programmes was the creation of opportunities for learning and experiencing new things. The participants identified these opportunities to include writing, painting, drawing, dancing, singing, surfing, life skills and homework: 'kids feel happy when a teacher teaches them new things' (Male, age 10). They also identified the ability to experience new places and activities outside of the community to be important, especially for children to develop a sense of happiness and hope for their future. The opportunity to participate infun activities was also identified to be a key component of an intervention programme. These activities included games, play, sports, art, music and the opportunity to move around safely.

Lastly, the participants mentioned the importance of programme interventions to assist children with the provision of their basic needs. They explained that when children do not have their basic needs met, they often feel bad about themselves or ashamed: 'When children do not have the things they need they feel bad about themselves, like when they do not have shoes for school' (Male, age 11). The participants identified their basic needs to include uniforms and supplies for school, food, a bed to sleep in and safety. 


\section{Discussion}

The current study aimed to explore children's perspectives on developing intervention programmes for the promotion of a healthy self-concept. The Children's Delphi technique was utilised within the child participation framework wherein children are recognised as the knowledgeable experts on matters relating to their lives. Through their engagement in the Delphi, the participants had the opportunity to represent a population (children in poverty) who are often excluded from the decision-making and planning processes around issues regarding their lives and their well-being. The participants identified the self-concept of children to be influenced by the children's reality, feelings and relationships. The participants' suggestions for intervention programmes included a focus on safety, social support, opportunities for learning and for fun and basic needs, all of which formed the ideal environment to nurture a healthy self-concept.

The children's reality was described by the participants in connection to their social relationships, spatial environment and their affective experiences. This reality often provided the participants with conflicting messages which challenged or distorted their self-integrity. This appeared to be the result of the inconsistent messages from their social and physical environment which became internalised as a means of self-understanding. This finding is in accordance with Cooley's 'looking glass self theory' (1902) which explains the self-concept to be shaped as a reflection of a person's social environment. The way an individual is acted upon by others becomes internalised as a means of knowing the self.

Another example of the conflicting messages was related to the physical infrastructure. While the participants mentioned beautiful spaces such as gardens to make children feel good about themselves, they also mentioned the dirty spaces in the community that contributed towards feelings of shame. The participants' experiences of violence in the outdoor spaces, where they felt children should be able to play, posed a barrier to their ability to form a meaningful place-identity within their community. The importance of place-identity on children's self-concept development is further supported by the research of Tuan (1997) and Ellis (2005). According to Tuan (1997), place is defined to be a source of security, meaning, belonging and identity which is facilitated through meaningful relationships made possible through bonds to a space. Ellis (2005) further described place to be a means through which people construct an identity. Although the children in this study were residing within conditions of poverty, this did not necessarily result in an unhealthy self-concept. Similarly, Proshansky and colleagues (1983) emphasised how living in a poor physical space does not necessarily contribute towards a negative place-identity if it leads to positive cognitions around a social context which are rewarding and positive for the individual. In the current study, the participants' reality within a space characterised by violence, allowed them to form meaningful relationships constructed around their strategies for survival, which led to a collective place-identity based on their unique experience as children within their communities.

It was further evident how the participants found ways of creating their own meaningful spaces within their communities, despite the lack of spaces formally designated for children. These spaces 
included community centres, schools, parks and beaches, and the homes of family and friends where the children created their own opportunities and spaces for play. The act of actively 'seeking out' and creating meaningful spaces formed a means through which they preserved their self-integrity within an environment which threatened their safety. This aligns with the research of Rogers et al. (2012) and Noble-Carr (2013) which supported the importance of meaning-making processes for nurturing a healthy self-concept in the face of adverse social and environmental conditions.

The participants' emphasis on play in supporting the way they thought and felt about themselves is consistent with current research and practice which supports play as essential for the social, emotional, cognitive and physical development and well-being of children (Gray, 2011, Milteer et al., 2012). Play has been shown to promote a sense of self-efficacy and self-control and to contribute towards emotional regulation, social intelligence and connectedness (Gray, 2011). For the participants in the current study, the combination of community violence, a lack of infrastructure for outdoor play (such as playgrounds and parks) and a lack of safe natural spaces (Adams \& Savahl, 2016) all limit their opportunities for play. It is axiomatic that programmes aimed at promoting a healthy self-concept should take into consideration the value of both structured activities which facilitate learning and unstructured forms of play, or in other words play which is structured by the children themselves (Milteer et al., 2012). Through unstructured play, children decide what they would like to do while simultaneously receiving the cognitive, social and emotional benefits which could also support the formation of a healthy self-concept (Milteer et al., 2012). Aligned with prior research on children's self-concept (see Demaray et al., 2009, McMuraay, 2010, Kenny \& McEachern, 2009), the availability of social support was identified to have an influence on the way the children thought and felt about themselves and consequently their behaviour. They identified family, teachers, peers and other adults in the community as sources of social support. The participants expressed a need for adults to be there to play with children, to talk to them about their problems, teach them new things and to keep them safe. They also explained the importance of having peers who were supportive when they had a problem. If the children were unable to find this support within a healthy and safe setting, they would seek this elsewhere, such as within a gang or amongst peers who participated in potentially harmful activities.

An additional recommendation provided by the participants was that intervention programmes should consider the provision of basicneeds. The children pointed out that the lack of basic material resources because of their impoverished living conditions contributed to feelings of shame, low selfworth and negatively affected their sense of dignity. The provision of basic material resources would form a buffer against these feelings of shame and would make the children feel valued because they would have the material necessities for their day to day survival, including clothes, food and supplies for school. The provision of basic needs and material resources has been found to be key aspects of children's well-being (September \& Savahl, 2009).

The critical finding of the study was the recommendation that intervention programmes must place the children's safety as a priority. This finding is especially relevant within the specific South African context as well as other communities throughout the world where high levels of community 
violence greatly affect children's opportunities for safety. The larger issue of community safety should be considered in combination with individual and organisational practices which promote a safe environment for children. The Delphi participants recommended addressing this issue through increasing adult supervision in spaces which could otherwise be dangerous for children and through the creation of more safe spaces within the community, such as after-school programmes and community centres.

\section{Conclusion}

The study provided a contribution towards an in-depth understanding of the challenges faced by children in their communities and towards the identification of potential solutions for promoting a healthy self-concept. The study further highlights the importance of engaging children in collaborative participatory research processes. An essential part of this process is delineating a clear strategy for how the research findings will translate into meaningful outcomes. It is recommended that the Children's Delphi technique be used for facilitating this process. An additional advantage of the use of the Children's Delphi in this study is that it appeared to benefit the self-concept of the participants, especially through engaging the young people in a meaningful way and through the development of new skills. In conclusion, the final recommendations for developing an intervention include:

1. Children's safety must be placed as a priority when it comes to the planning and execution of an intervention programme.

2. Social support in the form of caring family members, positive peer groups, older youth and other adults such as teachers and youth care workers should be provided.

3. Opportunities for learning and for fun should be equally available for children to develop their social skills.

4. Basic needs should be provided so that children can have access to the resources for their daily survival and to promote feelings of self-worth and social inclusion.

A key challenge of the children's Delphi was ensuring that the advice provided by the children was taken seriously when it came to the planning and implementation of intervention programmes. The collaboration with the local NGOs and schools assisted with this process. The findings were shared at parent-teacher meetings and provided a valuable contribution to the organisations' programme curriculum. Building trust with the participants through pro-longed engagement within the community further contributed towards creating an environment where the children felt comfortable to participate in an open and meaningful way. It is recommended that research focused on investigating various aspects of children's subjective well-being consider using the Children's Delphi technique as a methodological framework. It advances the notion that children are the authentic knowers and authoritative experts of their lives, offers a structured framework for the meaningful inclusion of children's views in research and thus aligns to Fattore, Mason \& Watson's (2012) epistemological position that children be located centrally in research that affects their lives. 


\section{References}

Adams, S. \& Savahl, S. (2016). Children's discourses of natural spaces: considerations for children's subjective well-being. Child Indicators Research. doi 10.1007/s12187016-9374-2

Benninger, E. (2016). Children's construction of the 'Self' within two impoverished urban communities in Cape Town. Unpublished Doctoral Dissertation. University of the Western Cape.

Benninger, E., \& Savahl, S. (2016a). Children's discursive constructions of the 'self'. Child Indicators Research, 1-29. doi:10.1007/s12187-016-9389-8

Benninger, E. \& Savahl, S. (2016b). The use of visual methods to explore how children construct and assign meaning to the "self" within two urban communities in the Western Cape, South Africa. International Journal of Qualitative Studies on Health and Well-being, 11, 31251-31264. doi: http://dx.doi.org/10.3402/ qhw.v11.31251

Casas, F. (2016). Children, adolescents and quality of life: the social sciences perspective over two decades. In F. Maggino (Ed.), A Life Devoted to Quality of Life (pp. 321). Cham: Springer International Publishing.

Crivello, G., Camfield, L. \& Woodhead, M. (2009). How can children tell us about their wellbeing? Exploring the potential of participatory research approaches within young lives. Social Indicators Research, 90(1), 51-72.

Demaray, M. K., Malecki, C. K., Rueger, S. Y., Brown, S. E. \& Summers, K. H. (2009). The role of youth's ratings of the importance of socially supportive behaviors in the relationship between social support and self-concept. Journal of Youth and Adolescence, 38(1), 13-28.

De Villiers, M. R., De Villiers, P. J. \& Kent, A. P. (2005). The Delphi technique in health sciences education research. Medical Teacher, 27(7), 639-643.

De Winter, M., Baerveldt, C., \& Kooistra, J. (1999). Enabling children: participation as a new perspective on child-health promotion. Child: Care, Health and Development, 25(1), 15-23.

Ellis, J. (2005). Place and identity for children in classrooms and schools. Journal of the Canadian Association for Curriculum Studies, 3, 55-73.

Fattore, T., Mason, J. \& Watson, E. (2007). Children's conceptualisation(s) of their wellbeing. Social Indicators Research, 80(1), 5-29.

Fattore, T., Mason, J. \& Watson, E. (2012). Locating the child centrally as subjects in research: towards a child interpretation of well-being. Child Indicators Research, $5,423-435$

Gray, P. (2011). The decline of play and the rise of psychopathology in children and adolescents. American Journal of Play, 3(4), 443-463.

Hanafin, S. \& Brooks, A. M. (2005). The Delphi Technique: A Methodology to Support the Development of a National set of Child Well-Being Indicators. Dublin: Department of child and youth affairs.

Hasson, F., Keeney, S. \& McKenna, H. (2000). Research guidelines for the Delphi survey technique. Journal of Advanced Nursing, 32(4), 1008-1015. 
Kennedy, H. P. (2004). Enhancing Delphi research: methods and results. Journal of Advanced Nursing, 45(5), 504-511.

Kenny, M. C. \& McEachern, A. (2009). Children's self-concept: a multicultural comparison. Professional School Counseling, 12(3), 207-212.

Langhout, R. D. \& Thomas, E. (2010). Imagining participatory action research in collaboration with children: an introduction. American Journal of Community Psychology, 46(1), 60-66.

Lockhat, R. \& Van Niekerk, A. (2000). South African children: a history of adversity, violence and trauma. Ethnicity and Health, 5(3-4), 291-302.

Mason, J. \& Watson, E. (2014). Researching children: research on, with, and by children. In A. Ben-Arieh, F. Casas, I. Frones \& J.

E. Korbin (Eds.), Handbook of Child Well-Being: Theories, Methods and Policies in Global Perspective (pp. 2757-2796). Dordrecht: Springer.

McMurray, I., Connolly, H. Preston-Shoot, M. \& Wigley, V. (2011). Shards of the old looking glass: restoring the significance of identity in promoting positive outcomes for looked-after children. Child \& Family Social Work, 16(2), 210-218.

Milteer, R. M., Ginsburg, K. R., Mulligan, D. A., Ameenuddin, N., Brown, A., Christakis, D. A. \& Swanson, W. S. (2012). The importance of play in promoting healthy child development and maintaining strong parent-child bond: focus on children in poverty. Pediatrics, 129(1), e204-e213.

Moore, T., Noble-Carr, D. \& McArthur, M. (2016). Changing things for the better: the use of children and young people's reference groups in social research. International Journal of Social Research Methodology. doi: 10.1080/ 13645579.2014.989640

Noble-Carr, D., Barker, J. \& McArthur, M. (2013). Me, Myself and I: Identity and Meaning in the Lives of Vulnerable Young People. Sydney: Institute of Child Protection Studies, Australian Catholic University.

Prilleltensky, I. (2010). Child wellness and social inclusion: values for action. American Journal of Community Psychology, 46(1-2), 238-249.

Proshansky, H. M., Fabian, A. K. \& Kaminoff, R. (1983). Place-identity: Physical world socialization of the self. Journal of Environmental Psychology, 3(1), 57-83.

Rogers, L. O., Zosuls, K. M., Halim, M. L., Ruble, D., Hughes, D. \& Fuligni, A. (2012). Meaning making in middle childhood: an exploration of the meaning of ethnic identity. Cultural Diversity and Ethnic Minority Psychology, 18(2), 99-108.

Savahl, S., Malcolm, C., Slembrouk, S., Adams, S., Willenberg, I. A. \& September, R. (2015). Discourses on well-being, Child Indicators Research. doi 10.1007/s12187-0149272-4

September, R. \& Savahl, S. (2009). Children's perspectives on child well-being. The Social Work Practioner-Researcher, 21(1), 23-40.

Shattell, M., Hamilton, D., Starr, S., Jenkins, J., Hinderliter, A. (2008). Mental health service needs of a Latino population: a community-based participatory research project. Issues in Mental Health Nursing, 29(4), 351. doi:10.1080/ 01612840801904316 
Staub, E. (2003). Notes on cultures of violence, cultures of caring and peace, and the fulfillment of basic human needs. Political Psychology, 24(1), 1-21.

Swann Jr, W. B., Chang-Schneider, C. \& Larsen McClarty, K. (2007). Do people's selfviews matter? Self-concept and self-esteem in everyday life. American Psychologist, 62(2), 84-94.

Tuan, Y.F. (1977). Space and Place: The Perspective of Experience. London: Edward Arnold. United Nations General Assembly (1989). Convention on the Rights of the Child. New York. Accessed 01/01/2016 from http://www.worldbank.org/en/country/southafrica/overview 